\title{
Sonographic Findings of Adductor Insertion Avulsion Syndrome With Magnetic Resonance Imaging Correlation
}

Jennifer S. Weaver, MD, Jon A. Jacobson, MD,

David A. Jamadar, MBBS, Curtis W. Hayes, MD

\section{Abbreviations}

AIAS, adductor insertion avulsion syndrome; MR, magnetic resonance; $T E$, echo time; TR, repetition time
Received December 10, 2002, from the Department of Radiology, University of Michigan Medical Center, Ann Arbor, Michigan USA. Manuscript accepted for publication December 18, 2002.

Address correspondence and reprint requests to Jon A. Jacobson, MD, Department of Radiology, University of Michigan Medical Center, 1500 E Medical Center Dr, TC 2910, Ann Arbor, MI 481090326 USA.

\section{Case Report}

Institutional Review Board approval was not required for this study. Our patient was a 19-year-old female cheerleader and soccer player who had a 1-month history of thigh pain that was accentuated with running and relieved with rest. Radiographs of the femur showed smooth, mature periosteal bone formation of the posteromedial midfemoral diaphysis (Fig. 2A).

Sonography was performed (by a fellowship-trained musculoskeletal radiologist with 5 years of experience in musculoskeletal sonography) 5 weeks after onset of symptoms with a 7-MHz linear transducer (HDI 3000; Philips Medical Systems, Bothell, WA) to evaluate thigh pain. Liberal acoustic transmission gel was used in place of a standoff pad. Sonographic findings included cortical irregularity surrounded by a hypoechoic area along the posteromedial midfemoral diaphysis (Fig. 2, B and C). 
Figure 1. Illustration of the posterior left femur showing insertion sites of the adductor muscles.

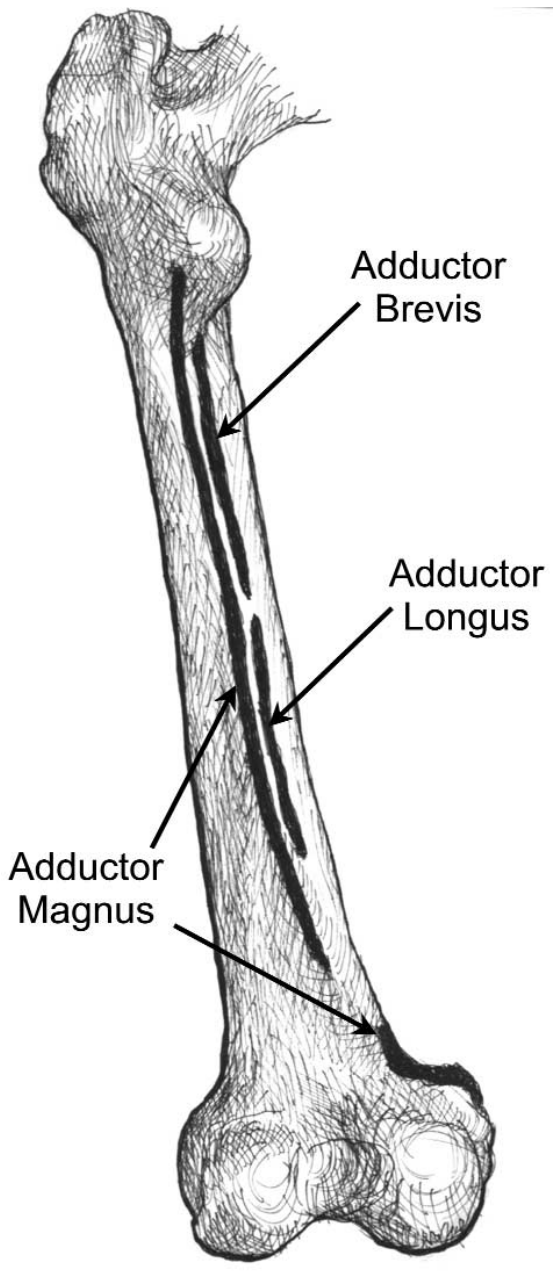

Figure 2. Images from a 19-year-old woman with AIAS A, Anteroposterior radiograph of the right femur showing a smooth, mature periosteal bone formation (open arrow) of the medial femoral cortex. B and C (opposite page), Axial (B) and sagittal (C) sonograms of the posteromedial midfemoral diaphysis (F) showing cortical irregularity (arrow) with surrounding hypoechogenicity (open arrows). D (opposite page), Axial color Doppler sonogram showing hyperemia (arrow) along the cortex of the femur (F). $\mathbf{E}$ and $\mathbf{F}$ (opposite page), Coronal T2-weighted (TR, 6000 milliseconds; TE, 84 milliseconds) fast spin echo fat saturation MR image (E) and axial T1-weighted (TR, 130 milliseconds; $T E, 1.7$ milliseconds) gradient echo fat saturation $M R$ image after intravenous gadolinium administration (F) showing enhancing bone marrow edema (curved arrow), adjacent enhancing periostitis (open arrow), and a fracture line (arrow).

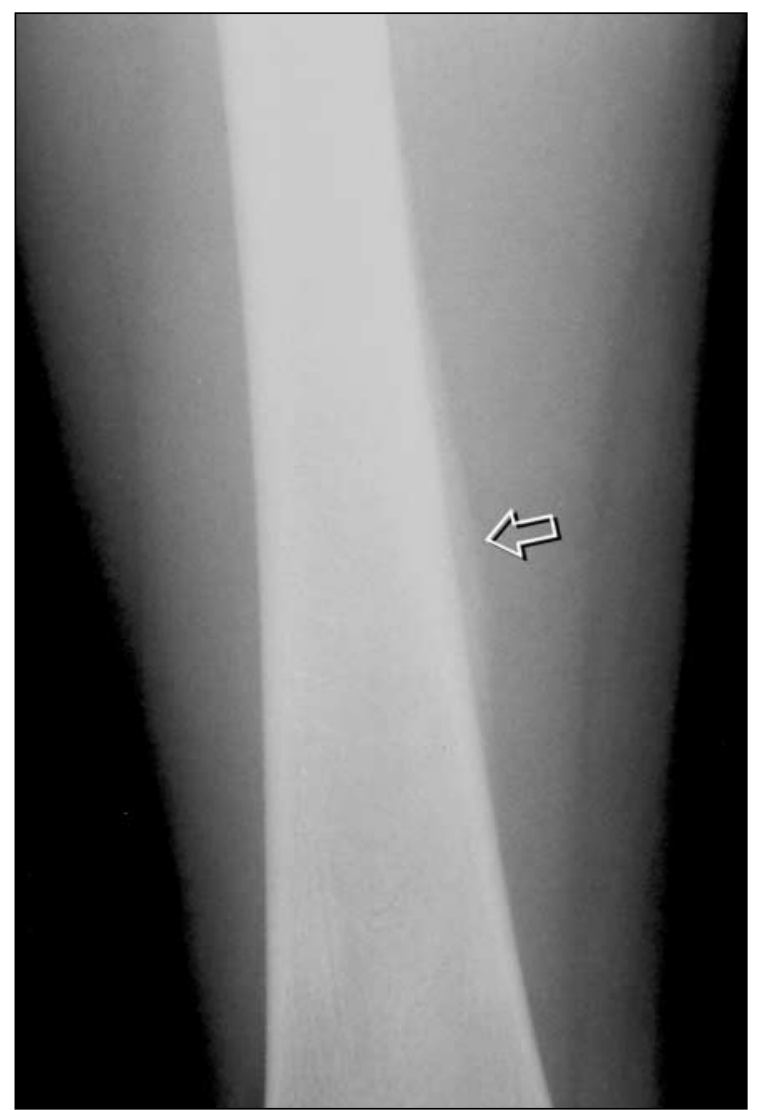

A

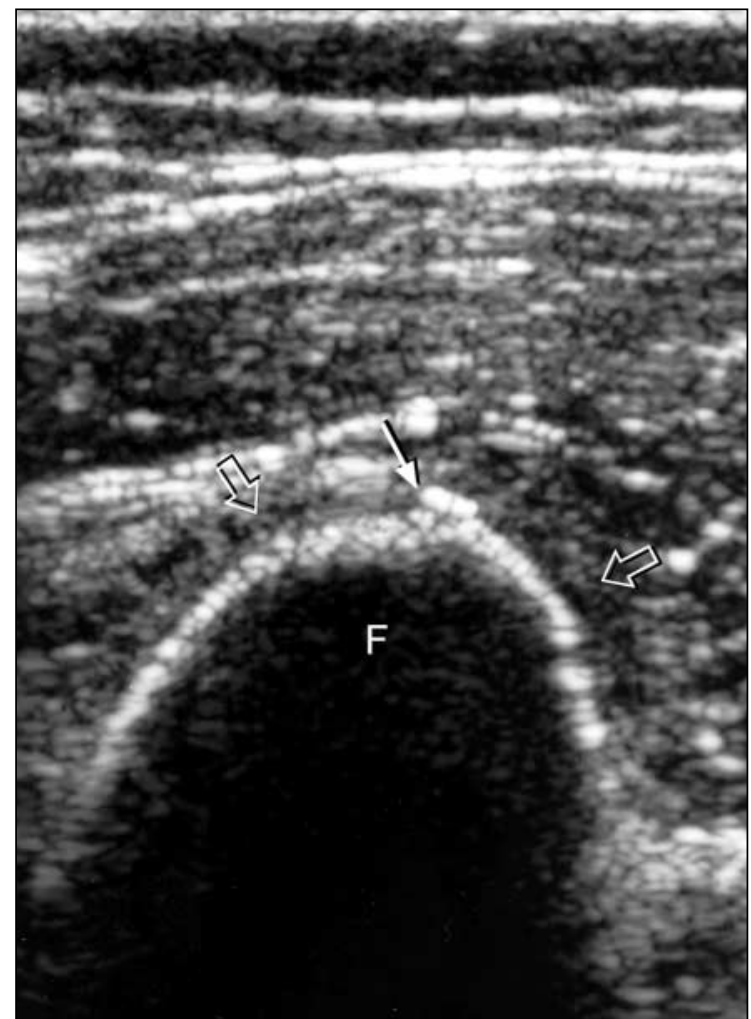

B 
Hyperemia was present on color Doppler imaging (Fig. 2D), and the patient had point tenderness in response to transducer pressure in this area.

Subsequent MR imaging (Signa; GE Medical Systems, Waukesha, WI) performed 3 weeks later
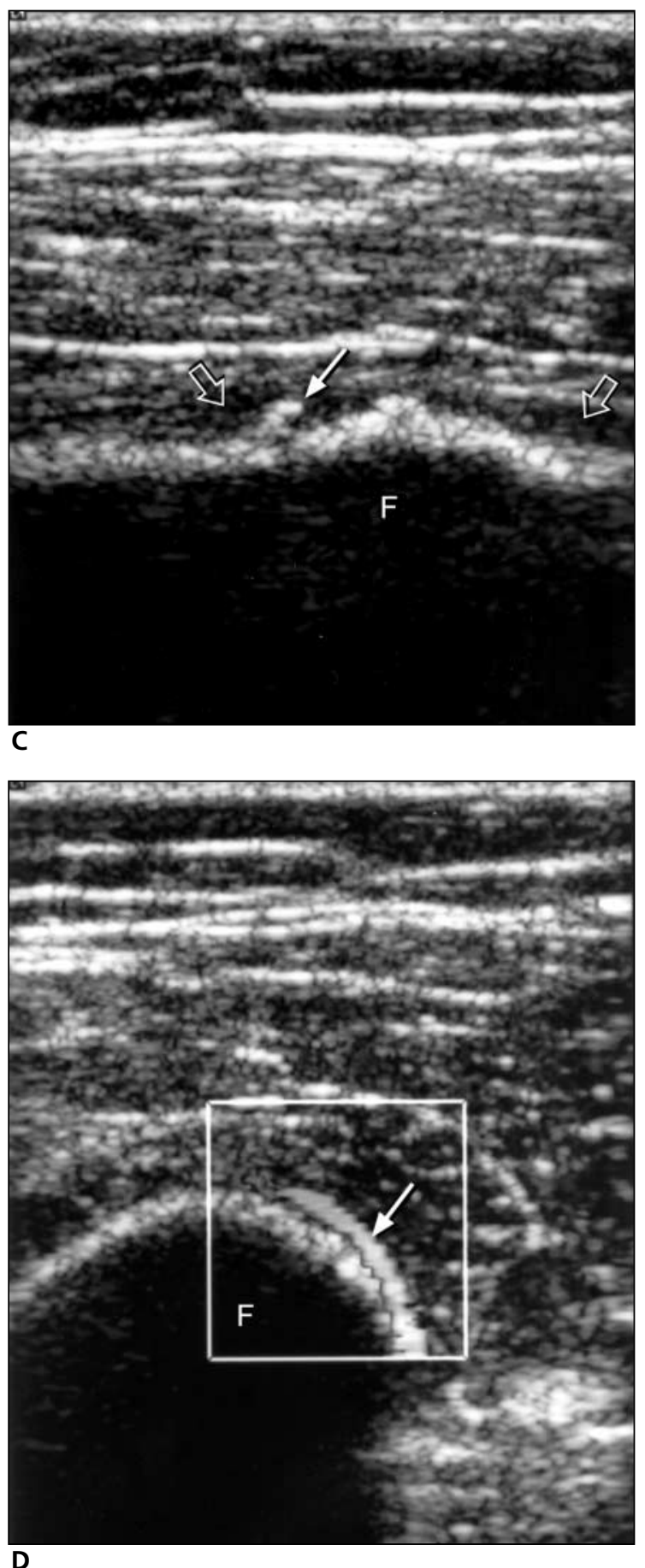

showed an increased signal in the bone marrow and soft tissues immediately adjacent to the femoral cortex on T2-weighted images (Fig. 2E; repetition time [TR], 6000 milliseconds; echo time [TE], 84 milliseconds; fast spin echo fat saturation; field of view, $36 \mathrm{~cm}$; slice thickness,

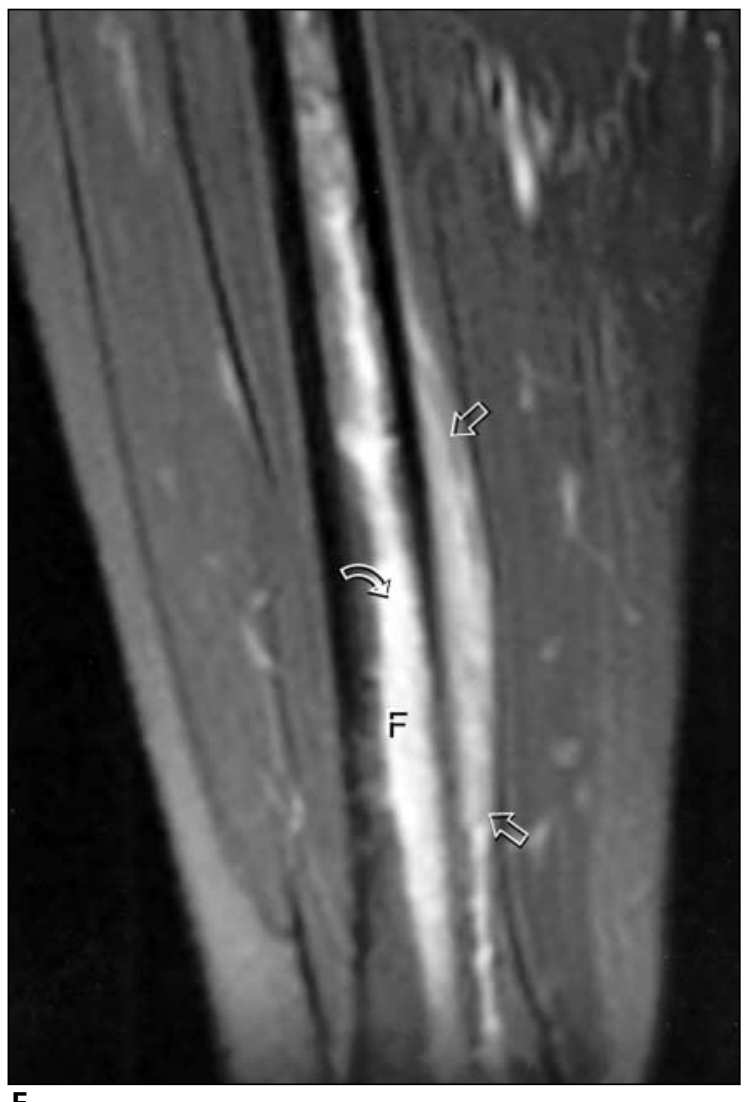

$\mathbf{E}$

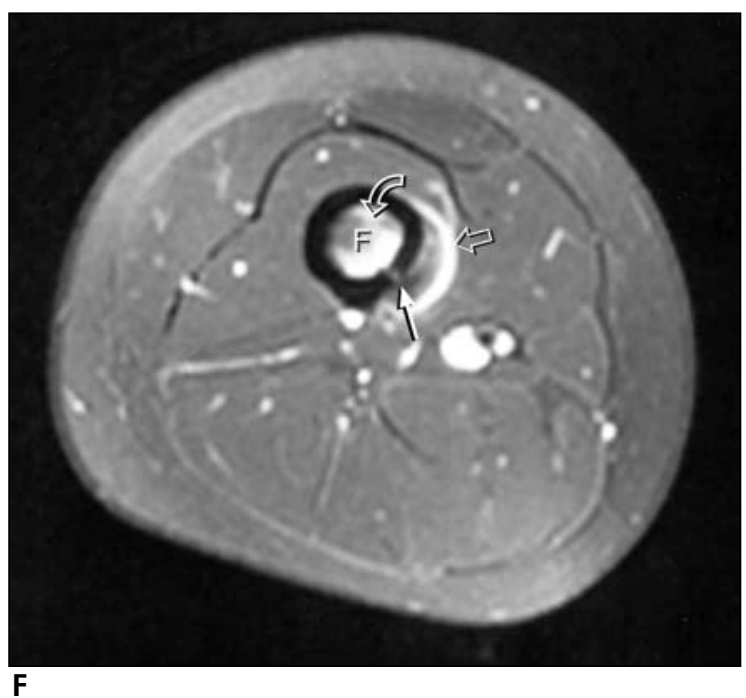


$5 \mathrm{~mm}$; slice gap, $1 \mathrm{~mm}$; $256 \times 256$ matrix, and 2 excitations) with enhancement after intravenous gadolinium administration on T1-weighted images (Fig. 2F; TR, 130 milliseconds; TE, 1.7 milliseconds; gradient echo fat saturation; field of view, $24 \mathrm{~cm}$; slice thickness, $5 \mathrm{~mm}$; slice gap, $1 \mathrm{~mm}$; $256 \times 192$ matrix; and 2 excitations). A linear area of increased signal intensity within the posteromedial femoral cortex was also present (Fig. 2F). This linear signal abnormality did not represent a normal vascular channel, which was visible on more cephalad images. The MR imaging findings were interpreted as enhancing bone marrow edema, periostitis, and fracture of the midfemoral diaphysis at the insertion site of the adductor musculature. These findings correlated with the sonographic findings.

The patient's symptoms resolved completely with conservative treatment and rest, and she has remained asymptomatic.

\section{Discussion}

Adductor insertion avulsion syndrome is a painful injury caused by repetitive avulsive stress trauma of the adductor muscles at their site of insertion along the posterior midfemoral diaphysis (Fig. 1). ${ }^{1-5}$ The repetitive stress results in traction periostitis, bone remodeling, and possible stress fracture. ${ }^{1,2}$ Symptoms appear after strenuous physical activity, and patients will have vague thigh, hip, and groin pain that is relieved with rest. This syndrome is most commonly associated with athletes and military personnel and is more common in female military recruits. ${ }^{2}$ It has been proposed that this is due to accentuated adductor strain in the relatively short female basic trainee compared with her male counterpart when adhering to the regulation 30-in marching step. ${ }^{7}$

The 3 adductor muscles of the proximal leg include the adductor brevis, longus, and magnus. The adductor brevis inserts proximally, whereas the adductor longus inserts along the posterior middle femur at the linea aspera (Fig. 1). The adductor magnus inserts along the posterior femur distally and at the adductor tubercle. Abnormalities at the proximal aspect of the femoral shaft suggest involvement of the adductor brevis, whereas abnormalities of the midfemoral shaft and posterior femur suggest adductor longus and adductor magnus involvement, respectively. ${ }^{2}$
The findings associated with AIAS on MR imaging have been described and include enhancing periostitis (related to traction periostitis or an adjacent osseous abnormality), bone marrow edema (from osseous stress reaction or fracture), and an increased linear intracortical signal, which represents a fracture line (Fig. 2, $\mathrm{E}$ and F). ${ }^{1,2}$ Nuclear bone scintigraphy shows increased radiopharmaceutical tracer uptake along the injury site, which is often visible before radiographic abnormalities are identified. ${ }^{1-5}$ Radiographs often initially show no abnormalities but may later show smooth, mature periosteal proliferation and possible stress fracture (Fig. 2A). ${ }^{1}$

Our results showed sonographic findings of cortical irregularity, adjacent abnormal hypoechogenicity and hyperemia, and pain in response to transducer pressure at the expected insertion of the adductor musculature along the posteromedial midfemoral diaphysis (Fig. 2, B-D). On the basis of the corresponding MR images, the cortical irregularity in our case represents visualization of the cortical fracture. The adjacent hypoechoic soft tissues could represent edema, hemorrhage, and periostitis, although hyperemia on color Doppler imaging and enhancement on MR images favor the latter.

The differential diagnosis includes infection and neoplasms. These conditions often show bone destruction and associated soft tissue masses. The clinical history and location of findings and symptoms are important in differentiating AIAS from infection and malignancy. An additional differential diagnosis consideration is an osteoid osteoma. Although smooth periostitis may appear similar to ASIS, visualization of the nidus of an osteoid osteoma on MR imaging, computed tomography, or radiography would help in this distinction.

One of the benefits of sonography is the ability to compare the affected extremity with the contralateral asymptomatic extremity; subtle sonographic abnormalities then become more conspicuous. In addition, the site of injury can often be found by having the patient indicate the site of symptoms. When a finding is identified on sonography, transducer pressure can be used to elicit symptoms, which aid in confirming the site of injury.

In the appropriate clinical setting, the sonographic findings of cortical irregularity and adjacent hypoechoic soft tissue at the posterior 
midfemoral diaphysis and point tenderness in response to transducer pressure at the injury site can suggest AIAS. It is important to consider this entity in patients with thigh pain, so that prompt treatment may avoid the development of stress fracture.

\section{References}

1. Anderson MW, Kaplan PA, Dussault RG. Adductor insertion avulsion syndrome (thigh splints): spectrum of MR imaging features. AJR Am J Roentgenol 2001; 177:673-675.

2. Anderson SE, Johnston JO, O'Donnell RO, Steinbach LS. MR imaging of sports-related pseudotumor in children: mid femoral diaphyseal periostitis at insertion site of adductor musculature. AJR Am J Roentgenol 2001; 176:1227-1231.

3. Charkes ND, Siddhivarn N, Schneck CD. Bone scanning in the adductor insertion avulsion syndrome ("thigh splints"). J Nucl Med 1987; 28:1835-1838.

4. Civelek AC, Scott WW Jr, Urban BA, Lietman SA. Thigh splints in a young female soccer player. Clin Nucl Med 2001; 26:341-344.

5. Singh AK, Dickinson C, Dworkin HJ, Sagar P, Patel S, Shirkhoda A. Adductor insertion avulsion syndrome. Clin Nucl Med 2001; 26:709-711.

6. Jacobson JA. Ultrasound in sports medicine. Radiol Clin North Am 2002; 40:363-386.

7. Ozburn MS, Nichols JW. Pubic ramus and adductor insertion stress fractures in female basic trainees. Mil Med 1981; 146:332-334. 